\title{
Computed tomography imaging of ovarian peritoneal carcinomatosis: a pictorial review
}

\author{
Binit Sureka ${ }^{A, B, E, F}$, Virendra Meena ${ }^{B, E, F}$, Pawan Garg ${ }^{B, F}$, Taruna Yadav ${ }^{E, F}$, Pushpinder Singh Khera ${ }^{A, F}$ \\ All India Institute of Medical Sciences, Jodhpur, India
}

\begin{abstract}
Gynaecological malignancies are the most common malignancies in women and also an important public health issue. In developing countries, there is a paucity of screening facilities and cancer awareness, so patients present at an advanced stage of disease, which severely limits the prognosis and clinical outcome. Among the gynaecological malignancies, ovarian malignancy has the second highest incidence in women according to the incidences. Ovarian malignancy is usually diagnosed at the advanced stages, and to improve the patient's survival, debulking surgery is very important. Advanced-stage disease is treated with either debulking surgery followed by adjuvant chemotherapy or initial neoadjuvant chemotherapy followed by debulking surgery. Imaging is very important in patient selection, in determining who will benefit from neoadjuvant chemotherapy just before debulking surgery. This article highlights the role of computed tomography (CT) in the detection of patterns of spread of ovarian malignancy, important for staging and management.
\end{abstract}

Key words: ovary, cancer, carcinomatosis, pathways, spread.

\section{Introduction}

Ovarian cancer is the fifth most common malignancy among women worldwide and the second most common gynaecological malignancy [1]. Ovarian malignancy is usually diagnosed at advanced stage of the disease with five-year survival rate in stage III disease being $37 \%$ and stage IV disease being 24\% [2]. The International Federation of Gynaecology and Obstetrics (FIGO) ovarian cancer staging system was first published in 1973 and was revised in 1988 and 2014. Table 1 and Figure 1 summarise the new FIGO 2014 staging of ovarian cancer $[3,4]$.

The treatment planning of advanced ovarian malignancies includes both primary debulking surgery followed by adjuvant chemotherapy, and if non-resectable disease is found on preoperative imaging, neoadjuvant chemotherapy followed by debulking surgery. Several recent randomised control trials clearly show similar disease-free survival rates between stage III or stage IV disease in patients who initially underwent chemotherapy followed by debulking surgery [5].

Detection of peritoneal disease on preoperative imaging is very difficult, and interpretation of imaging findings requires in-depth knowledge of complex peritoneal anatomy, peritoneal fluid flow direction, and specific anatomic locations where debulking surgery is technically difficult. The presence of disease at these sites is very helpful for surgeons for planning of surgery.

This article describes the role of radiologists in planning the management of advanced ovarian malignancies. Radiologists provide essential information about routes of disease dissemination and common sites for intra- and extraperitoneal disease. Preoperative imaging is very helpful in detection of sites of potentially non-resectable disease or resectable disease, which is very helpful in the planning of debulking surgery. Early signs of peritoneal spread of

Correspondence address:

Prof. Binit Sureka, Diagnostic and Interventional Radiology, All India Institute of Medical Sciences, Basni Industrial Area, 342005, Jodhpur, India,

e-mail: binitsurekapgi@gmail.com

Authors' contribution:

A Study design · B Data collection · C Statistical analysis · D Data interpretation - E Manuscript preparation · F Literature search · G Funds collection 
ovarian malignancy may be missed if subtle deposits are not detected. Precise interpretation of ovarian malignancy spread on computed tomography is necessary for good quality reporting of ovarian malignancies for optimal management of disease.

Table 1. FIGO staging of ovarian cancer $2014[3,4]$

\begin{tabular}{|c|c|}
\hline $\begin{array}{l}\text { Stage I } \\
\text { Tumour confined to ovaries }\end{array}$ & $\begin{array}{l}\text { IA: Tumour limited to one ovary or fallopian tube, capsule intact, no tumour on surface, no tumour } \\
\text { cells in ascites or washings } \\
\text { IB: Tumour involves both ovaries or fallopian tubes, otherwise like stage IA } \\
\text { IC: Tumour involves one or both ovaries with any of the following: capsule rupture, tumour on surface } \\
\text { of the ovary, positive peritoneal washings or ascites } \\
\text { IC1: Intraoperative spill } \\
\text { IC2: Capsule rupture before surgery or tumour on ovarian or fallopian tube surface } \\
\text { IC3: Malignant cells in the ascites or peritoneal washings }\end{array}$ \\
\hline $\begin{array}{l}\text { Stage II } \\
\text { Tumour involves one or both ovaries with } \\
\text { pelvic extension (below the pelvic brim) } \\
\text { or primary peritoneal cancer }\end{array}$ & $\begin{array}{l}\text { IIA: Extension and/or implant on uterus and/or fallopian tubes } \\
\text { IIB: Extension to other pelvic intraperitoneal tissues }\end{array}$ \\
\hline $\begin{array}{l}\text { Stage III } \\
\text { Tumour involves one or both ovaries with } \\
\text { cytological or histologically confirmed spread } \\
\text { to the peritoneum outside the pelvis and/or } \\
\text { metastasis to the retroperitoneal lymph nodes }\end{array}$ & $\begin{array}{l}\text { IIIA: Positive retroperitoneal lymph nodes and /or microscopic metastasis beyond the pelvis } \\
\text { IIIA1: Positive retroperitoneal lymph nodes only } \\
\text { IIIA1(i) Metastasis } \leq 10 \mathrm{~mm} \\
\text { IIIA1(ii) Metastasis }>10 \mathrm{~mm} \\
\text { IIIA2: Microscopic, extrapelvic (above the brim) peritoneal involvement } \pm \text { positive retroperitoneal } \\
\quad \text { lymph nodes } \\
\text { IIIB: Macroscopic, extrapelvic, peritoneal metastasis } \leq 2 \mathrm{~cm} \pm \text { positive retroperitoneal lymph nodes. } \\
\text { Includes extension to capsule of liver/spleen } \\
\text { IIIC: Macroscopic, extrapelvic, peritoneal metastasis }>2 \mathrm{~cm} \pm \text { positive retroperitoneal lymph nodes. } \\
\text { Includes extension to capsule of liver/spleen }\end{array}$ \\
\hline $\begin{array}{l}\text { Stage IV } \\
\text { Distant metastasis excluding peritoneal } \\
\text { metastasis }\end{array}$ & $\begin{array}{l}\text { IVA: Pleural effusion with positive cytology } \\
\text { IVB: Hepatic and/or splenic parenchymal metastasis, metastasis to extra-abdominal organs } \\
\text { (including inguinal lymph nodes and lymph nodes outside of the abdominal cavity) }\end{array}$ \\
\hline
\end{tabular}

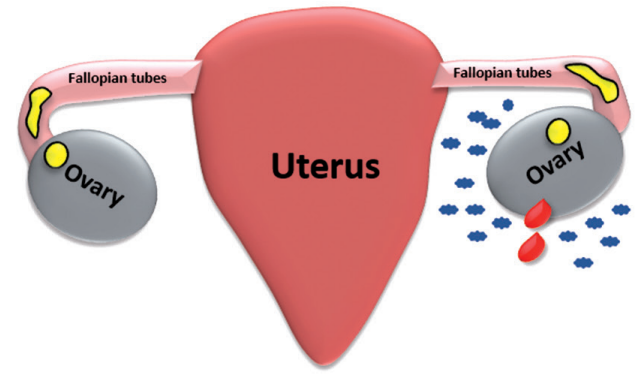

Stage IA - cancer limited to one ovary or fallopian tube (yellow) Stage IB - cancer limited to both ovaries or fallopian tubes Stage IC1 - cancer results from intraoperative spill (red) Stage IC3 - cancer cells are found in peritoneal washings (blue)

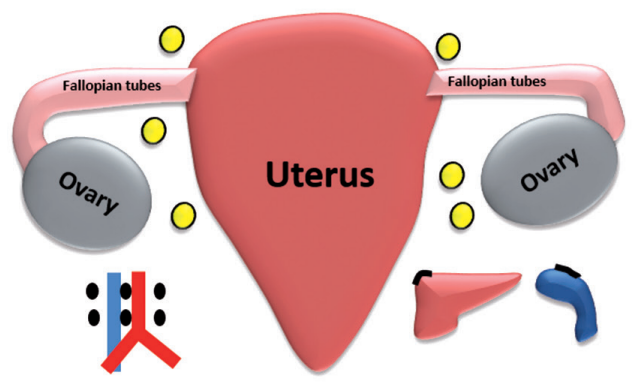

Stage III - retroperitoneal lymph nodes, peritoneal metastases, extension into capsule of liver or spleen
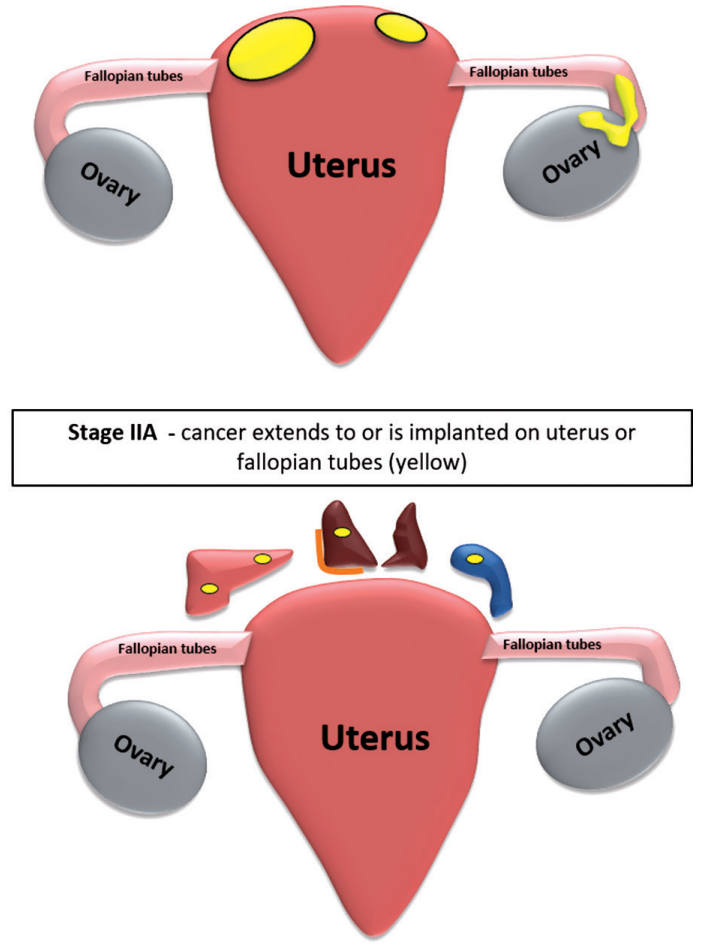

Stage IVA - Pleural effusion with positive cytology Stage IVB - Hepatic and/or splenic parenchymal metastasis

Figure 1. Drawing showing FIGO staging of ovarian cancer 


\section{Clinical and laboratory findings in ovarian cancer}

Early stage ovarian cancers are usually asymptomatic. Late-stage ovarian cancers, which account for approximately $60 \%$ of cases, often have nonspecific symptoms, not recognised as symptoms of cancer [6]. Nonspecific symptoms of ovarian cancer are back pain, fatigue, abdominal pain/bloating, constipation, or urinary symptoms.

In addition to these nonspecific symptoms, ovarian cancer may present with paraneoplastic syndromes such as subacute cerebellar degeneration; sudden onset of seborrheic keratoses (Leser-Trélat sign); or unexplained spontaneous, recurrent, or migratory venous thrombotic events (Trousseau syndrome). Advanced disease may present with symptoms of regional spread or metastasis, such as bowel or ureteral obstruction, or shortness of breath $[7,8]$. Sex cord-stromal tumours can present early at stage I because of hormonal manifestations such as precocious puberty, abnormal uterine bleeding, and virilisation [9].

Cancer antigen (CA) 125 is the biomarker commonly tested for ovarian cancer. CA125 is elevated in about $80 \%$ of epithelial ovarian cancers overall, but in only $50 \%$ of early-stage epithelial ovarian cancers [8]. Biomarkers for nonepithelial ovarian cancers include inhibin A/B for sex cord-stromal tumours, and serum $\alpha$-fetoprotein and quantitative beta human chorionic gonadotropin for germ cell tumours [10]. Human epididymal protein 4 (HE4) is a relatively new tumour marker. Elevated serum HE4 levels have been shown to have higher sensitivity (89\%) and specificity (92\%) than CA125 for distinguishing ovarian cancer from benign ovarian disease in premenopausal women, particularly in early-stage disease. As well as these biomarkers, complete blood count, blood chemistry including liver function tests and calcium (to assess for paraneoplastic syndromes), and serum biomarkers should be obtained if ovarian cancer is suspected.

\section{Computed tomography technique}

The optimal abdominal and pelvic computed tomography (CT) technique for staging ovarian cancer includes water as an oral contrast media, and in the absence of contraindications to intravenous contrast media, the use of intravenous contrast media is mandatory. Opacification of the gastrointestinal tract with oral contrast media helps to distinguish between luminal lesions and serous or mesenteric deposits; however, small, calcified deposits may be masked by oral contrast [11]. At our institution, image acquisition is done at 70 seconds after injection of contrast media. Acquisition for an abdominal and pelvic CT extends from the lower thorax to the inguinal regions with reconstruction of images with an axial slice thickness of 1-2 mm [10]. To include chest or not is an institutional policy. At our institute, we routinely scan the whole chest to look for lung metastases.

\section{Routes of dissemination}

Ovarian cancer usually spreads by direct infiltration into surrounding pelvic organs. Ovarian malignancies most commonly involve the adjacent adnexal structures like the uterus, fallopian tube, and contralateral ovary. Pelvic organs like the rectum, sigmoid colon, urinary bladder, and pelvic side wall may also be invaded. Spread of ovarian malignancy outside the pelvis occurs by intraperitoneal seeding, lymphatic transmission, or haematogenous spread.

\section{Intraperitoneal seeding}

Intraperitoneal seeding is most common mode of spread of ovarian malignancy, and during laparotomy $70 \%$ patients have peritoneal metastasis. The ovary is covered by single layer of epithelium and in epithelial ovarian malignancy, tumour cells are detached from the epithelium and spread in peritoneal fluid. Tumour cells initially shed into the dependent aspect of the rectouterine pouch and then move into lateral paravesical region because of gravity, and then move in a cranial direction with circulating peritoneal fluid through the right paracolic gutter to the hepatorenal pouch, right subphrenic space and then inferiorly into the inframesocolic compartment (Figure 2). The rectouterine pouch, right lower quadrant, sigmoid colon, and right paracolic gutter are the most common sites for peritoneal deposits due to gravity-dependent areas in the peritoneal cavity [12]. Peritoneal deposits are most commonly seen in the right paracolic gutter rather than the left due to the presence of the phrenicocolic ligament on the left side.

\section{Lymphatic dissemination}

Ovaries have three main drainage pathways - paraaortic and paracaval nodes are most common sites of lymph node metastasis, and they are drained by lymphatic vessels that follow the ovarian veins. The second most common site of lymph node metastasis is along the external iliac and obturator lymph nodes along the broad ligament (Figure 3) and inguinal lymph nodes along the round ligament [13].

\section{Haematogeneous spread}

Haematogeneous spread is the least common mode of spread of ovarian malignancy. It often involves colon, liver, small intestine, and lung in decreasing order. Ovarian malignancy can rarely involve the spleen, kidney, and brain (Figure 4) [14].

\section{Sites of tumour dissemination}

\section{Peritoneal involvement}

Visible metastatic implants outside the pelvis and retroperitoneal lymphadenopathy are seen in $60 \%$ of epithelial 
A

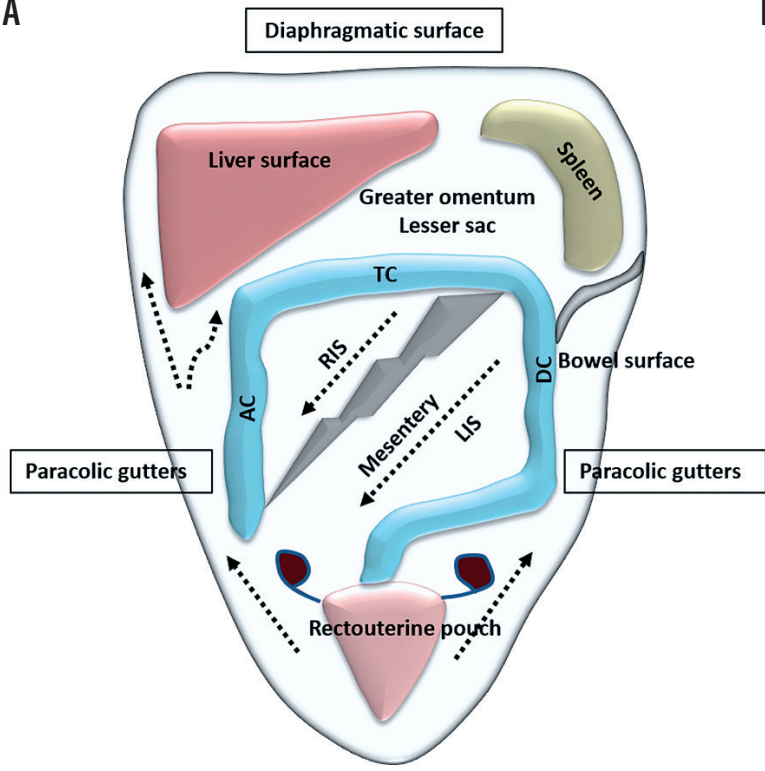

B Serous surfaces

Liver, spleen, subphrenic space, diaphragm, pleural cavity, bowel

Perihepatic space Gallbladder fossa

Falciform ligament Porta hepatis

Bilateral paracolic spaces Peritoneal and serous deposits

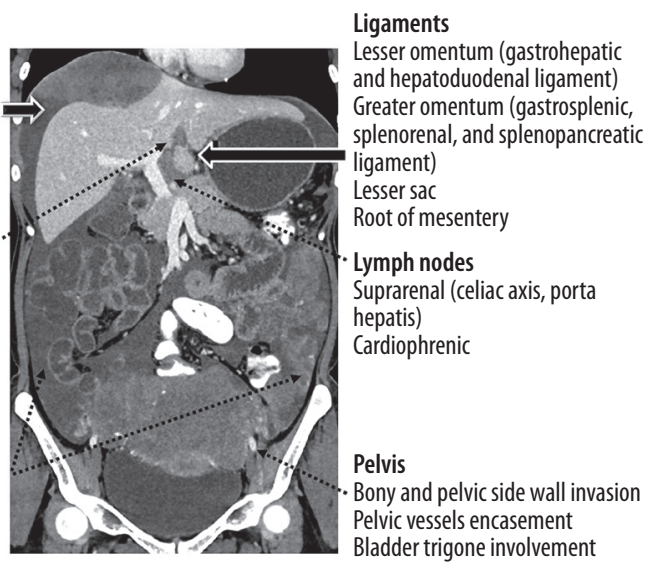

Figure 2. A) Drawing showing the peritoneal space anatomy and pathways of spread of tumour cells into the peritoneal cavity. B) Coronal contrast-enhanced CT showing tumoural deposits into the peritoneal cavity along the serous surface, perihepatic space, paracolic spaces, ligaments, lymph nodes, and pelvis
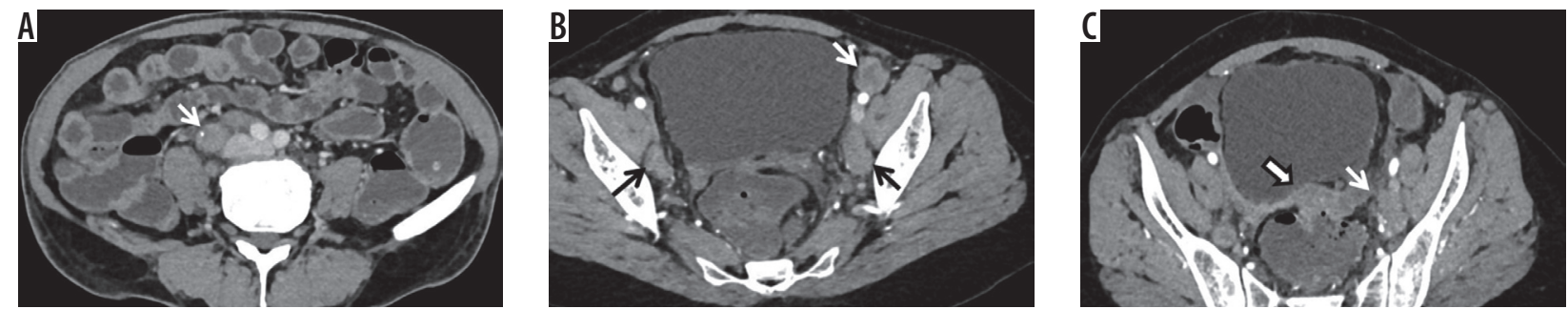

Figure 3. Lymphatic dissemination in a case of ovarian cancer: A) Contrast-enhanced CT images showing enlarged lymph node (white arrow) in right common iliac region with calcific foci; B) bilateral internal iliac (black arrow) and left external iliac group (white arrow); C) obturator group (white arrow) with a deposit in rectouterine pouch (arrowhead)
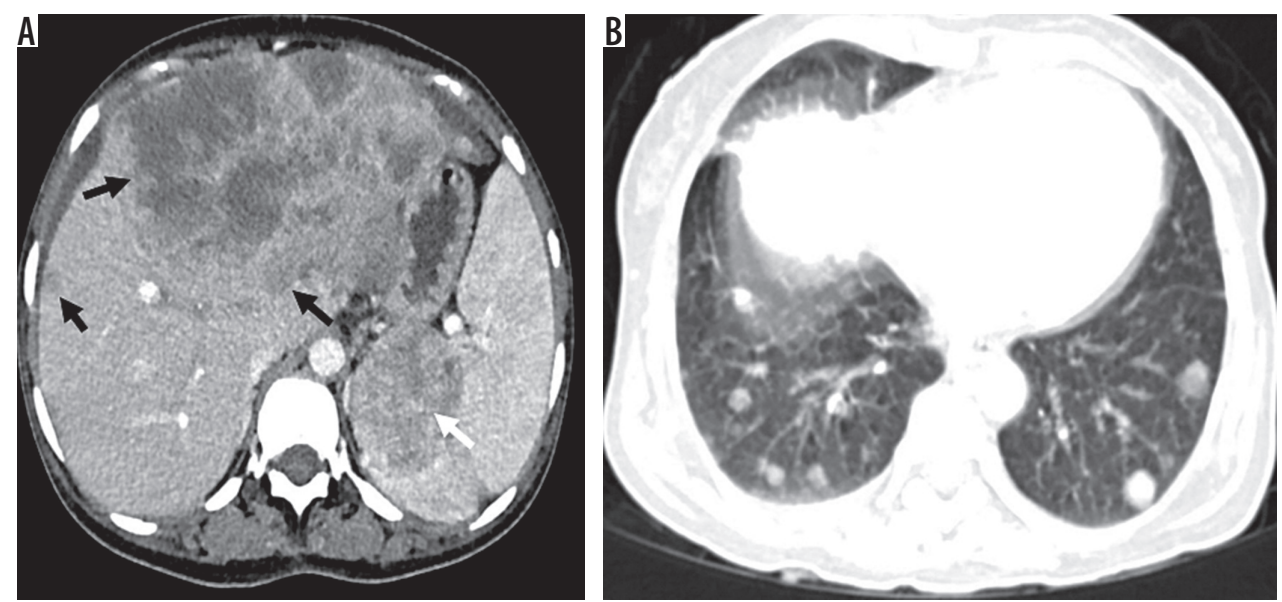

Figure 4. Haematogeneous spread from ovarian cancer: A) Contrast-enhanced CT images showing large metastatic deposit in liver (black arrow) and spleen (white arrow); B) and multiple metastatic nodules in bilateral lungs

ovarian malignancy [15]. Subtle thickening, nodularity, soft tissue in omental fat, peritoneal enhancement, and loculated ascites are subtle signs of peritoneal spread (Figure 5). Negative oral contrast is recommended for the detection of subtle serous deposits on the bowel surface.

Morphologically, there are three types of implants: solid, cystic, and mixed. In all three categories, calcifications may or may not be present. Different patterns of implants have been described:

- micronodular pattern - characterised by the presence of tiny 1-5-mm nodules involving the serosa and subserosal fat;

- nodular pattern - more than 5-mm-sized nodules, which are oval or round in shape with spiculated margins; 

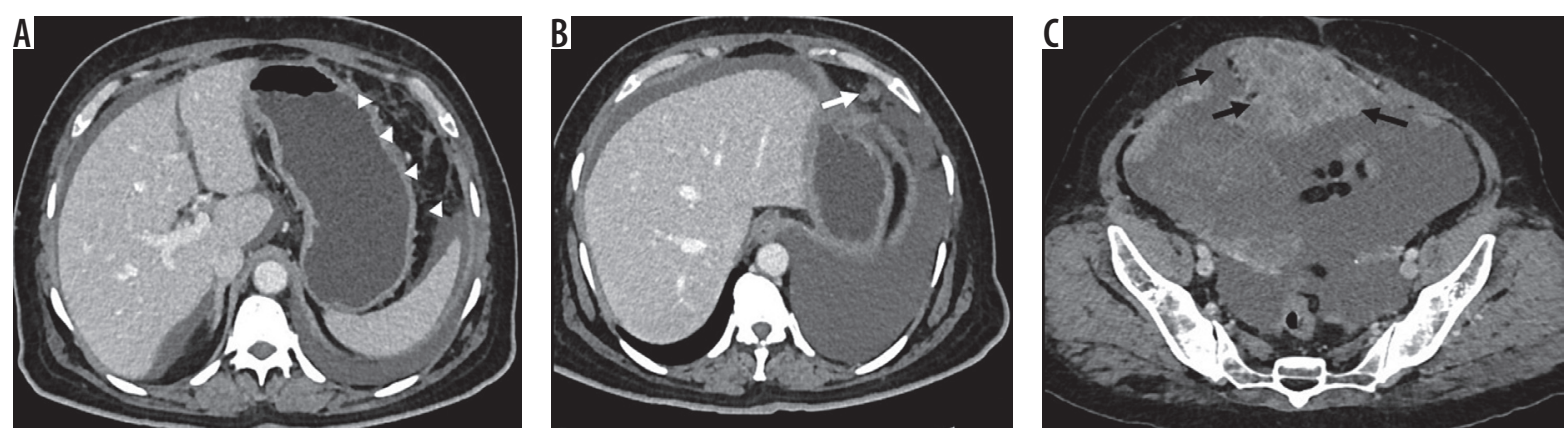

Figure 5. Contrast-enhanced computed tomography showing various types of peritoneal involvement in peritoneal carcinomatosis: A) subtle thickening and fat stranding; B) nodular pattern C); mass-like pattern with neoplastic ascites

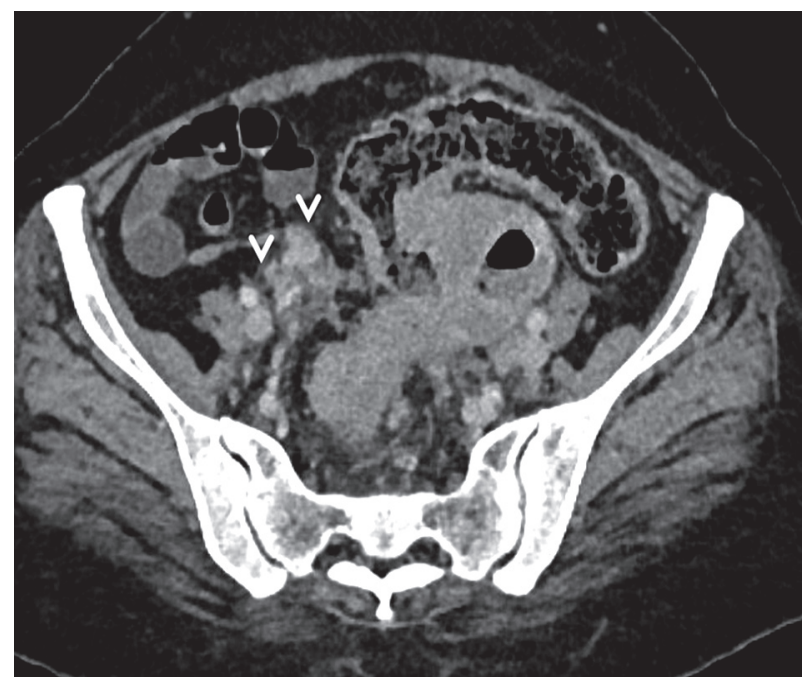

Figure 6. Contrast-enhanced computed tomography image showing nodular deposits (arrowheads) in mesentery

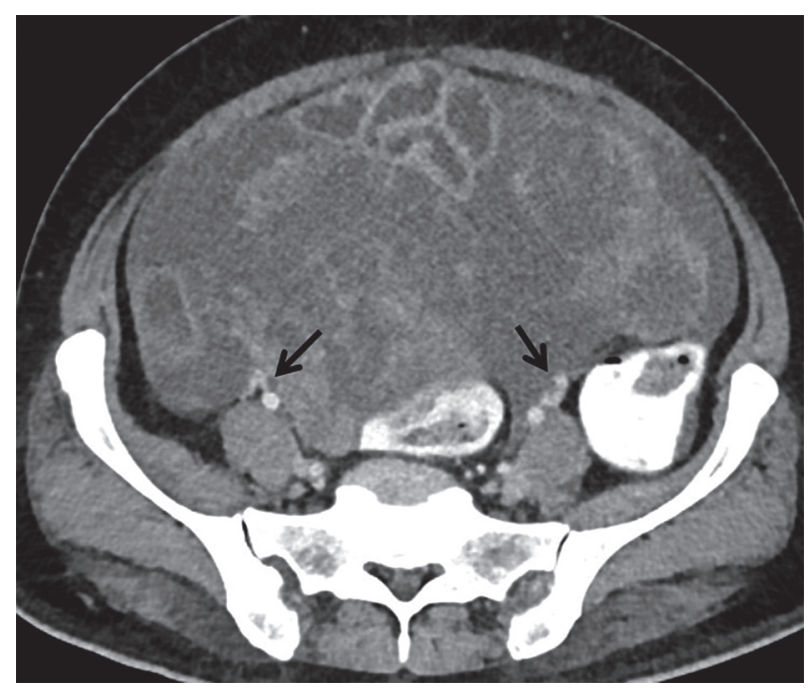

Figure 7. Pelvic involvement in ovarian cancer - contrast-enhanced computed tomography image showing tense ascites with plaque-like thickening of the peritoneum encasing the bilateral iliac vessels (arrows)

- plaque-like pattern - confluence of nodular implants giving a sheet-like pattern typically found in subdiaphragmatic spaces;
- mass-like pattern - similar to plaque-like pattern, commonly found in the pelvis;

- omental cake - diffuse involvement of greater omentum with fibrotic reaction leading to consolidation of omental fat;

- teca aspect or ileal freezing - sleeve-like covering of small bowel loops by thickened a layer of visceral peritoneum with or without bowel obstruction;

- calcified implants - nodular calcific deposits in peritoneum along with calcified lymph nodes, especially seen in serous ovarian adenocarcinoma;

- neoplastic ascites - free fluid in the peritoneal cavity may be due to increased capillary permeability and fluid production or blockage of lymphatics leading to decreased absorption.

\section{Mesentery}

Implantation of tumour cells in small bowel mesentery is seen usually surrounding the terminal ileum. Morphological appearances of mesenteric involvement can be in the form of plaque-like thickening, reticulonodular lesions, subtle soft tissue haziness, or a mass-like deposit (Figure 6).

\section{Lymph nodes}

The presence of enlarged suprarenal lymph nodes at the level of the celiac axis, porta hepatis, and cardiophrenic lymph nodes makes the disease prone to suboptimal resection, and patients should go for neoadjuvant chemotherapy before surgery [16].

\section{Pelvis}

Pelvic side wall invasion should be suspected when the mass lesion is within $3 \mathrm{~mm}$ of the pelvic side wall, or surrounds or distorts more than $90 \%$ of the circumference of iliac vessels (Figure 7) [17]. 

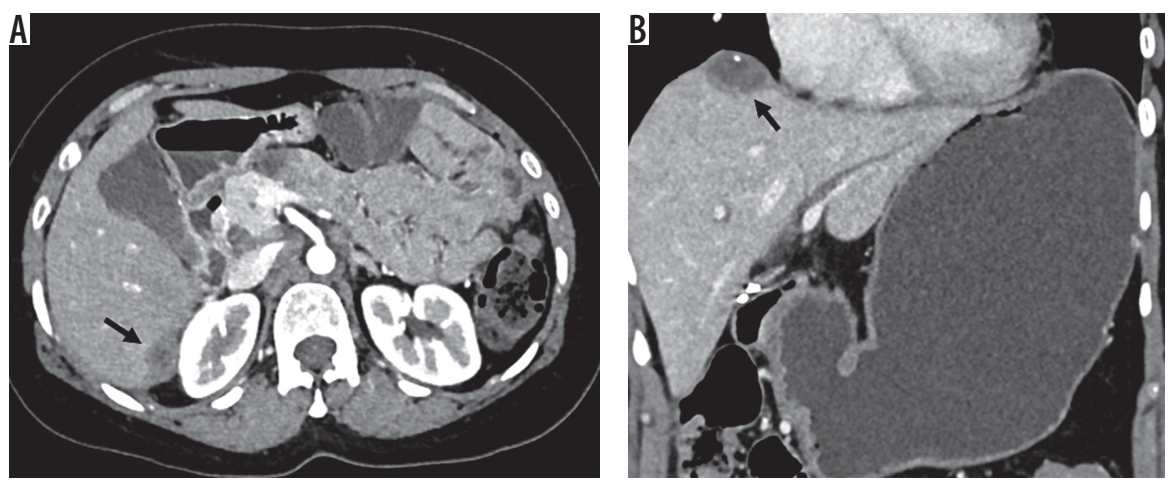

Figure 8. Subserosal deposits in ovarian cancer. Contrast-enhanced computed tomography images in two different patients showing subserosal implants in liver surface (arrow)

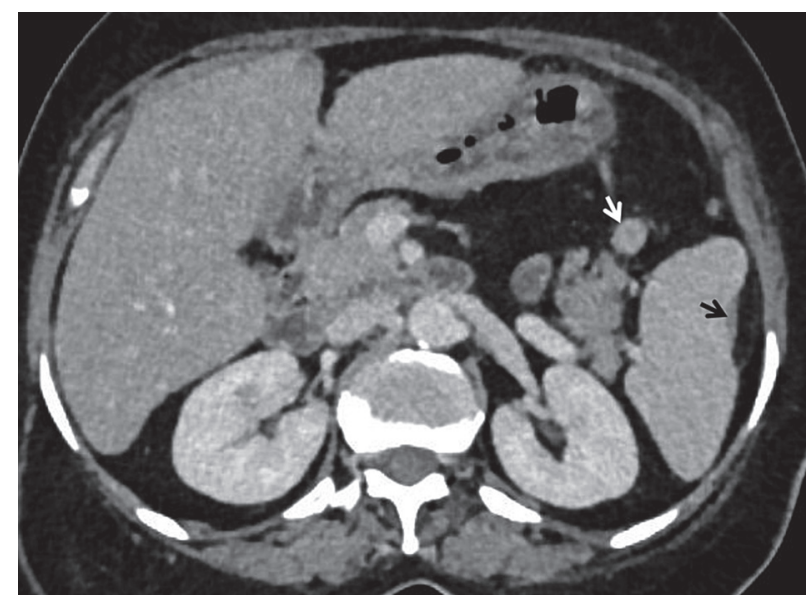

Figure 9. Subcapsular splenic deposit. Contrast-enhanced computed tomography image showing subcapsular splenic deposit (black arrow) and another nodular deposit in the gastrosplenic ligament (white arrow)

\section{Visceral surfaces}

\section{Liver}

The location, site, and size of liver implants should be documented in the report. Deposits in the region of Morrison's pouch, inferior vena cava, and around the right hepatic vein must be documented, because deposits in this region can cause excessive haemorrhage at the time of surgery. Secondary invasion of surface or subserosal implant (Figure 8) into the liver parenchyma should be well documented in the report [16].

\section{Spleen}

Subcapsular splenic deposits and parenchymal metastases have to be differentiated because this allows the surgeon to decide between splenectomy and spleen-sparing surgery (Figure 9) [17].

\section{Subphrenic space}

Right subphrenic involvement is very common because of preferential flow of peritoneal fluid from the right para-

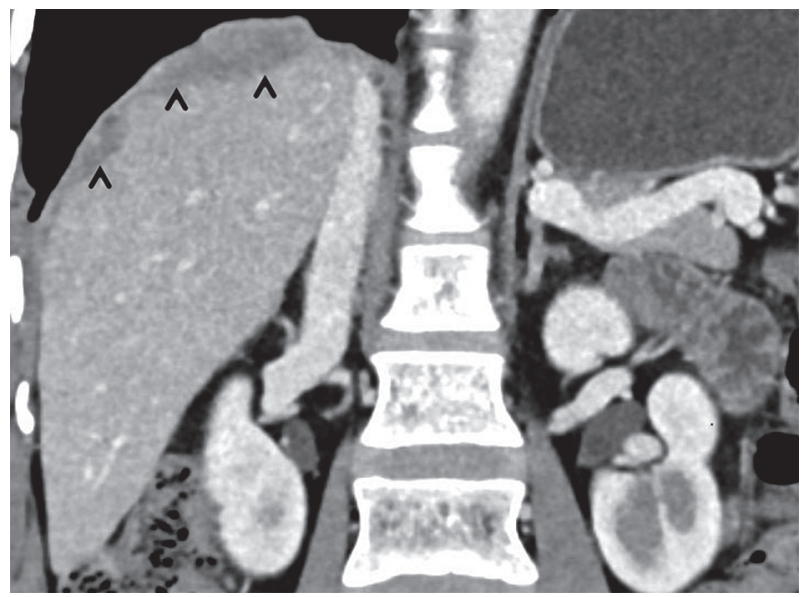

Figure 10. Subphrenic deposit in ovarian cancer. Coronal contrast-enhanced computed tomography image showing nodular thickening in right subphrenic region (arrowheads) indenting the liver surface

colic gutter. Involvement of the subphrenic region is best visualised in coronal and sagittal sections of post-contrast CT images. Imaging features of sub phrenic involvement include the presence of nodules, masses, thickening, and enhancement of the hemi diaphragm. Finding of subphrenic involvement must be written in radiologists' reports because sub phrenic involvements usually require upper-quadrant peritonectomy (Figure 10) [18,19].

\section{Bowel}

Magnetic resonance imaging (MRI) is superior to CT for assessment of bowel involvement in ovarian malignancies. Imaging findings of bowel involvement in ovarian malignancy include segmental or focal bowel wall thickening, focal nodules, serous infiltrations, or any definitive mass. Extensive bowel resections are not recommended due to functional complications (Figure 11) [20-22].

\section{Pleural cavity}

The presence of pleural effusion must be documented, although this finding is not specific unless tumour cells are detected in the fluid cytology. 


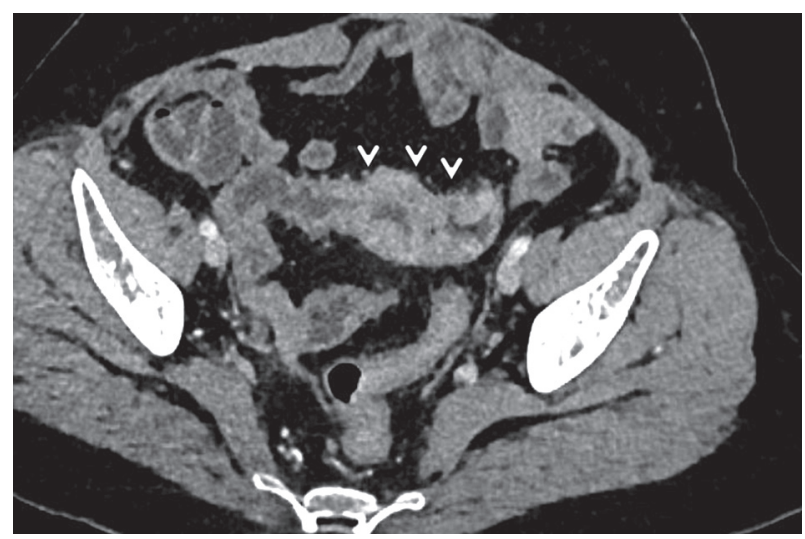

Figure 11. Bowel involvement in ovarian cancer. Contrast-enhanced computed tomography image showing symmetrical nodular thickening (arrowheads) of the ileal loops in a case of ovarian cancer

\section{Ligaments}

\section{Greater omentum}

Imaging features of involvement of greater omentum range from infiltrative fat stranding to discrete and confluent masses forming omental cake. Involvement of transverse mesocolon also has to be commented upon because a modified surgical approach is required for the same.

\section{Lesser omentum}

The lesser omentum is a part of the peritoneum, which suspends the lesser curvature of the stomach, and it is composed of gastro-hepatic ligament and hepatoduodenal ligament. The left gastric artery, vein, and gastro-hepatic lymph nodes are contents of the gastro hepatic ligament, and it is recognised by identification of the left gastric artery on post-contrast CT images. The portal vein, common bile duct, hepatic artery, and portocaval lymph nodes are content of the hepatoduodenal ligament. Subtle soft tissues in the lesser omentum, and nodules and lymphadenopathy in the gastro hepatic and hepatoduodenal ligaments must be written in radiology reports. Involvement of the lesser omentum precludes surgery because of the

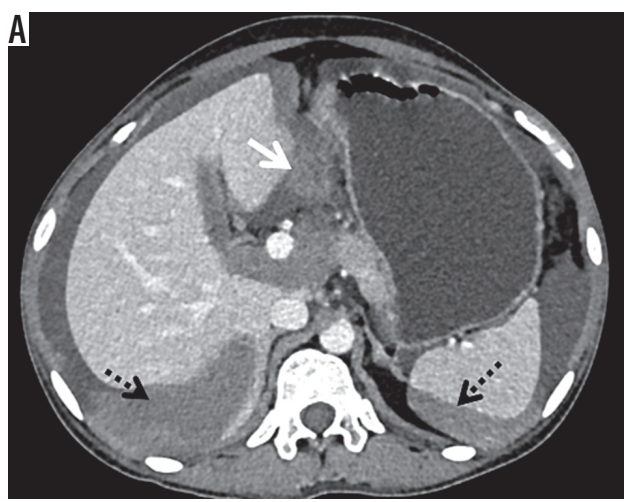

complicated anatomy of the lesser omentum, which poses significant surgical challenges (Figure 12) $[18,19]$.

\section{Lesser sac}

The lesser sac is a potential space between the stomach and pancreas. The lesser sac on the left side is outlined by the splenorenal ligament, gastrosplenic ligament, and splenopancreatic ligament. The lesser sac, on the right side, connects with the greater sac through the foramen of Winslow. Involvement of the lesser sac must be included in the radiological report because its involvement makes ovarian malignancy unresectable. Thickening, nodule, mass, fat stranding, and the presence of fluid in the lesser sac are considered to be signs of disease involvement (Figure 13).

\section{Perihepatic space}

The gallbladder fossa and fissure for the falciform ligament are the main components of the perihepatic space. Involvement of the falciform ligament should be looked for carefully in multiple planes to differentiate from liver parenchymal lesions. The falciform ligament, gallbladder fossa and periportal space should be carefully evaluated, because deposits more than $2 \mathrm{~cm}$ in size are predictors of non-optimal debulking (Figure 14) [23].

\section{Differential diagnosis and computed tomography mimics of peritoneal carcinomatosis}

Various pathologies involving the peritoneum can mimic peritoneal carcinomatosis. Table 2 summarises the mimics of peritoneal carcinomatosis and their imaging features [24-33].

\section{Comparison between computed tomography and magnetic resonance imaging in the detection of peritoneal implants}

CT is the imaging modality of choice for staging ovarian cancer. However, CT is limited in detecting small perito-

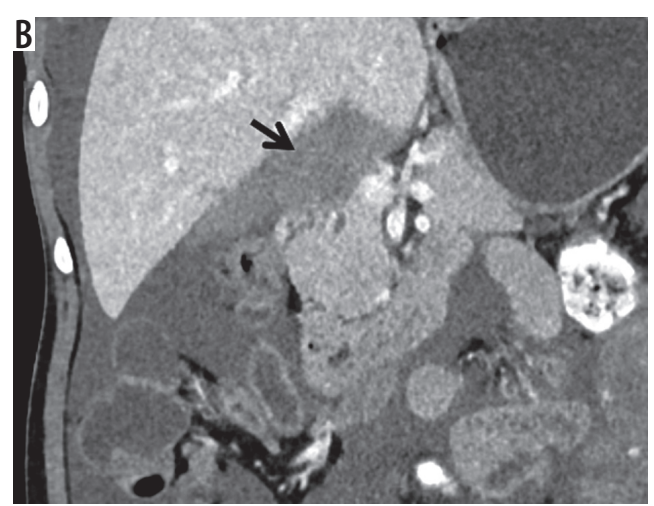

Figure 12. Lesser omentum involvement in ovarian cancer. Axial and coronal CT images showing soft tissue deposits in gastrohepatic ligament (white arrow) and in hepatoduodenal ligament (black arrow) of lesser omentum region. Perisplenic and perihepatic enhancing deposits (dashed arrows) with neoplastic ascites also evident 


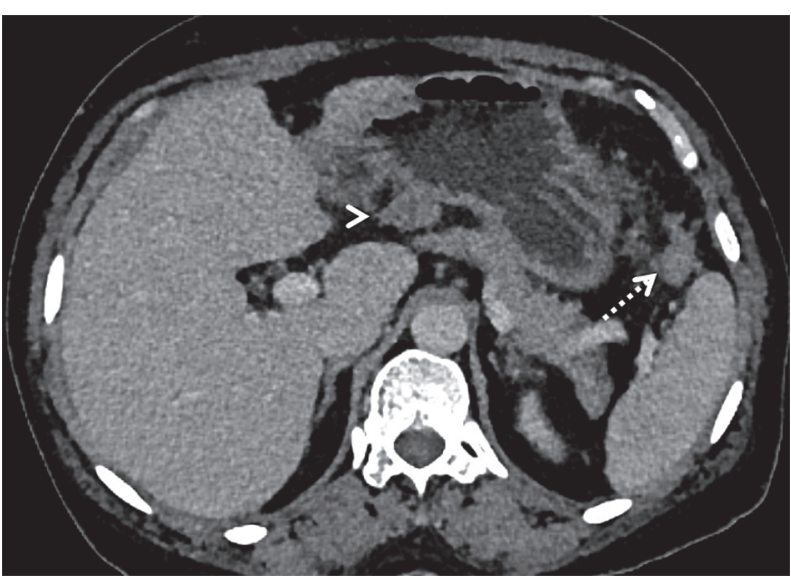

Figure 13. Lesser sac deposit in ovarian cancer. Contrast-enhanced computed tomography image showing metastatic deposits in between pancreas and stomach (arrowhead) and in gastrosplenic ligament (dashed arrow)

neal metastases. The sensitivity and specificity of CT in detecting tumour implants larger than $1 \mathrm{~cm}$ range from $85-93 \%$ and $91-96 \%$, respectively, but the sensitivity decreases to $25-50 \%$ in detecting implants that are $1 \mathrm{~cm}$ or smaller. MRI combining delayed gadolinium-enhanced imaging and diffusion-weighted MR imaging (DWM$\mathrm{RI}$ ) is more accurate than CT for estimating peritoneal tumour burden [34]. Low et al. [35] have shown that MRI is superior to CT in estimating peritoneal cancer index, with sensitivity $95 \%$, specificity $70 \%$, and accuracy $88 \%$,
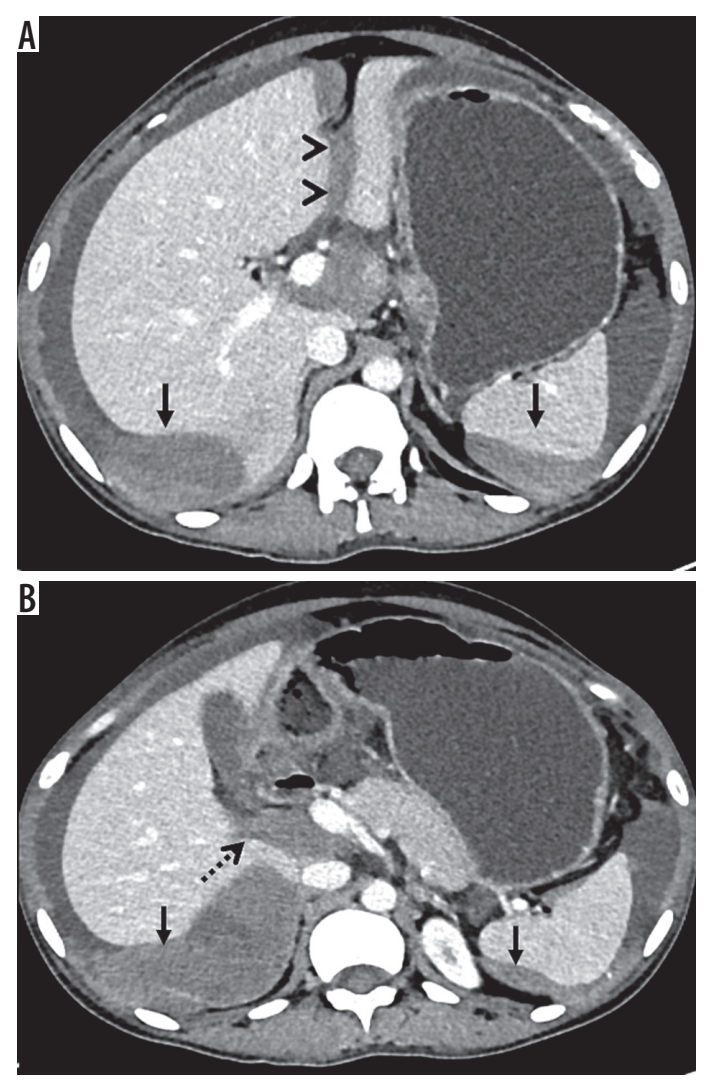

Figure 14. Perihepatic deposits in ovarian cancer. Axial contrast-enhanced computed tomography images showing: A) deposits in the falciform ligament (arrowheads), periportal region; B) around gallbladder fossa (dashed arrow) with perihepatic and perisplenic enhancing deposits (arrows)

Table 2. Differential diagnosis and computed tomography mimics of peritoneal carcinomatosis

\begin{tabular}{|c|c|}
\hline Carcinomatosis mimics & Imaging features \\
\hline Tuberculous peritonitis & $\begin{array}{l}\text { Three types - wet, fixed fibrotic, and dry plastic types. Young age, presence of free or loculated ascites, necrotic lymph } \\
\text { nodes, matted bowel loops, and smooth peritoneal thickening confirms the diagnosis [24] }\end{array}$ \\
\hline $\begin{array}{l}\text { Peritoneal } \\
\text { lymphomatosis }\end{array}$ & $\begin{array}{l}\text { Associated findings of splenomegaly, aneurysmal dilatation of bowel loop segment, confluent homogeneously enhancing } \\
\text { non-necrotic lymph nodes encasing the vessels producing a'sandwich sign' [25] }\end{array}$ \\
\hline $\begin{array}{l}\text { Primary peritoneal } \\
\text { mesothelioma }\end{array}$ & $\begin{array}{l}\text { Older age of presentation, strong association of asbestos exposure, two imaging patterns - nodular mass like large confluent } \\
\text { masses and diffuse nodular thickening of peritoneum, no calcification, ascites (loculated or diffuse) with omental caking [26] }\end{array}$ \\
\hline Pseudomyxoma peritonei & Gelatinous ascites causing classical scalloping of the surface of abdominal organs [27] \\
\hline Peritoneal sarcomatosis & $\begin{array}{l}\text { Associated with gastrointestinal stromal tumour, liposarcoma, and leiomyosarcoma. Distinguishing imaging features include } \\
\text { heterogeneous bulky masses, hypervascularity with or without hemoperitoneum and variable presence of ascites [28] }\end{array}$ \\
\hline $\begin{array}{l}\text { Desmoplastic small } \\
\text { round cell tumour }\end{array}$ & $\begin{array}{l}\text { Rare aggressive malignancy that occurs in adolescents and young adults, mostly male, and carries a poor prognosis. } \\
\text { Imaging features are characterised by single or multiple peritoneal masses in the omentum or paravesical region, } \\
\text { which spread later to the entire abdominal cavity [29] }\end{array}$ \\
\hline Splenosis & $\begin{array}{l}\text { Spontaneous auto-transplantation of splenic tissue after traumatic injury or splenectomy, }{ }^{99 \mathrm{~m}} \text { Tcechnetium-labelled RBC } \\
\text { scintigraphy with SPECT/CT imaging is the modality of choice for diagnosis [30] }\end{array}$ \\
\hline Melanosis & $\begin{array}{l}\text { Unusual condition characterised by either focal or diffuse brown or black pigmentation of the peritoneum, usually } \\
\text { surgico-pathological diagnosis; associated with various causes like metastatic melanoma, mature ovarian cystic teratoma, } \\
\text { ovarian serous cystadenoma, enteric duplication cyst, peritoneal cyst [31] }\end{array}$ \\
\hline Sclerosing peritonitis & $\begin{array}{l}\text { Chronic inflammatory condition of the peritoneum with an unknown aetiology, eventually progresses to sclerosis and } \\
\text { membrane formation with subsequent cocoon formation. Imaging features are central accumulation of the small } \\
\text { intestine encased by a dense membrane, intestinal obstruction, ascites, calcification bowel serous surface [32] }\end{array}$ \\
\hline Omental infarct & Fatty, large (> $5 \mathrm{~cm}$ ) encapsulated mass, with soft-tissue stranding adjacent to the ascending colon [33] \\
\hline Fat saponification & $\begin{array}{l}\text { Sequelae of pancreatitis resulting in mesenteric and retroperitoneal fat necrosis. Imaging shows scattered tiny soft tissue } \\
\text { density nodules and fluid in vicinity of inflammation [33] }\end{array}$ \\
\hline
\end{tabular}


Potentially nonresectable disease

Extensive involvement of small bowel or mesenteric root Lymph node above celiac axis

Pleural involvement

Pelvic sidewall invasion

Bladder trigone involvement

Intrahepatic metastases

Metastatic implants near right hepatic vein

Implants $>2 \mathrm{~cm}$ in diameter in diaphragm, lesser sac,

porta hepatis, gallbladder fossa, falciform ligament,

gastrosplenic or gastrohepatic ligament

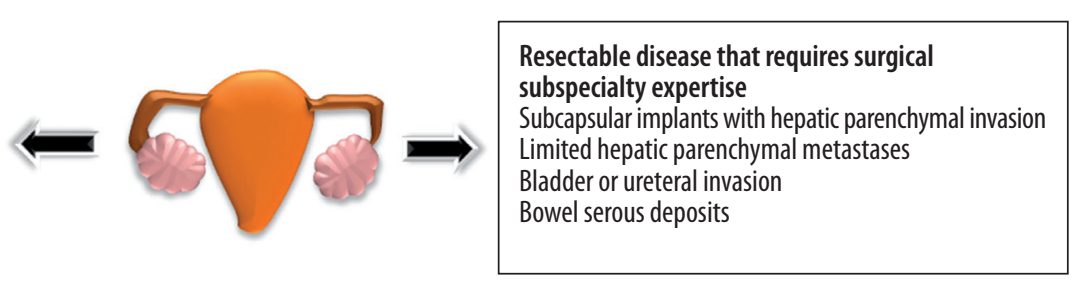

Figure 15. Diagram showing resectable and non-resectable parameters of ovarian cancer

compared to CT, which had sensitivity $55 \%$, specificity $86 \%$, and accuracy $63 \%$. DWMRI with a high b-value (e.g. $800 \mathrm{~s} / \mathrm{mm}^{2}$ ) increases the specificity in peritoneal carcinomatosis detection because of better suppression of the bowel contents, and the combination of DW-MRI and contrast-enhanced MRI further improve the detection accuracy [36]. For treatment response evaluation, functional imaging with DWMRI or PET-CT is recommended. Postoperative inflammation and oedema would result in high ADC, in contrast to tumours that have low ADC, allowing for differentiation of disease recurrence from postoperative fibrosis and inflammation. DWMRI is superior to PET-CT in detecting perihepatic and serous deposits because of physiological uptake in the liver and bowel and respiratory motion interference [37]. PET-CT is a helpful imaging adjunct in the presence of rising tumour markers but equivocal CT or MRI, in detecting disease recurrence in ovarian cancer [38].

\section{Conclusions}

Accurate peritoneal anatomy and knowledge of imaging features of peritoneal carcinomatosis is essential for appropriate management and care of patents with ovarian cancer. It is important to be aware of the various resectable and nonresectable parameters of ovarian cancer (Figure 15). CT is the most widely used imaging modality for assessment; however, MR with advanced functional tools can be helpful in assessing the tumour response to treatment.

\section{Acknowledgements}

The authors want to thank Dr. Shashank Shekhar, Additional Professor, Department of Obstetrics \& Gynaecology, AIIMS Jodhpur, for referring cases of gynaecological malignancies.

\section{Conflict of interest}

The authors report no conflicts of interest.

\section{References}

1. Permuth-Wey J, Sellers TA. Epidemiology of ovarian cancer. Methods Mol Biol 2009; 472: 413-437.

2. Jemal A, Siegel R, Ward E, et al. Cancer statistics, 2009. CA Cancer J Clin 2009; 59: 225-249.

3. Prat J; FIGO Committee on Gynecologic Oncology. FIGO Guidelines. Staging classification for cancer of the ovary, fallopian tube, and peritoneum. Int J Gynaecol Obstet 2014; 124: 1-5.

4. Javadi S, Ganeshan DM, Qayyum A, et al. Ovarian cancer, the revised FIGO staging system, and the role of imaging. AJR Am J Roentgenol 2016; 206: 1351-1360.

5. Vergote I, Tropé CG, Amant F, et al. Neoadjuvant chemotherapy or primary surgery in stage IIIC or IV ovarian cancer. N Engl J Med 2010; 363: 943-953

6. National Institutes of Health. National Cancer Institute. Surveillance, Epidemiology, and End Results Program. Statistical summaries: cancer stat fact sheets (ovary) and cancer statistics review (CSR), 1975-2013. Available at: http:// seer.cancer.gov/statistics/ summaries.html (Accessed: 27.04.2016).
7. Tavassoli FA, Devilee P (eds.). Pathology and genetics of tumours of the breast and female genital organs. International Agency for Research on Cancer, Lyon 2003.

8. Cannistra SA. Cancer of the ovary. N Engl J Med 2004; 351: 25192529. Review. Erratum in: N Engl J Med 2005; 352: 104.

9. Zhang M, Cheung MK, Shin JY, et al. Prognostic factors responsible for survival in sex cord stromal tumors of the ovary - an analysis of 376 women. Gynecol Oncol 2007; 104: 396-400.

10. Doubeni CA, Doubeni AR, Myers AE. Diagnosis and management of ovarian cancer. Am Fam Physician 2016; 93: 937-944.

11. Pannu HK, Bristow RE, Montz FJ, et al. Multidetector CT of peritoneal carcinomatosis from ovarian cancer. RadioGraphics 2003; 23: 687-701.

12. Coakley FV, Hricak H. Imaging of peritoneal and mesenteric disease: key concepts for the clinical radiologist. Clin Radiol 1999; 54: 563-574.

13. Feldman GB, Knapp RC. Lymphatic drainage of the peritoneal cavity and its significance in ovarian cancer. Am J Obstet Gynecol 1974; 119: 991-994. 
14. Rose PG, Piver MS, Tsukada Y, Lau TS. Metastatic patterns in histologic variants of ovarian cancer: an autopsy study. Cancer 1989; 64: 1508-1513.

15. Heintz AP, Odicino F, Maisonneuve P, et al. Carcinoma of the ovary. FIGO 26th Annual Report on the Results of Treatment in Gynecological Cancer. Int J Gynaecol Obstet 2006; 95: S161-S192.

16. Akin O, Sala E, Moskowitz CS, et al. Perihepatic metastases from ovarian cancer: sensitivity and specificity of CT for the detection of metastases with and those without liver parenchymal invasion. Radiology 2008; 248: 511-517.

17. Nougaret S, Addley HC, Colombo PE, et al. Ovarian carcinomatosis: how the radiologist can help plan the surgical approach. Radiographics 2012; 32: 1775-1800.

18. Sugarbaker PH. Peritonectomy procedures. Surg Oncol Clin N Am 2003; 12: 703-727.

19. Sugarbaker PH. Surgical responsibilities in the management of peritoneal carcinomatosis. J Surg Oncol 2010; 101: 713-724.

20. Colombo PE, Mourregot A, Fabbro M, et al. Aggressive surgical strategies in advanced ovarian cancer: a monocentric study of 203 stage IIIC and IV patients. Eur J Surg Oncol 2009; 35: 135-143.

21. Dvoretsky PM, Richards KA, Angel C, et al. Survival time, causes of death, and tumor/ treatment-related morbidity in 100 women with ovarian cancer. Hum Pathol 1988; 19: 1273-1279.

22. Dvoretsky PM, Richards KA, Angel C, et al. Distribution of disease at autopsy in 100 women with ovarian cancer. Hum Pathol 1988; 19: 57-63.

23. Forstner R, Sala E, Kinkel K, Spencer JA. ESUR guidelines: ovarian cancer staging and follow-up. Eur Radiol 2010; 20: 2773-2780.

24. Akhan O, Pringot J. Imaging of abdominal tuberculosis. Eur Radiol 2002; 12: 312-323.

25. Horger M, Müller-Schimpfle M, Yirkin I, et al. Extensive peritoneal and omental lymphomatosis with raised CA 125 mimicking carcinomatosis: CT and intraoperative findings. Br J Radiol 2004; 77: 71-73.

26. Levy AD, Arnáiz J, Shaw JC, et al. From the archives of the AFIP: primary peritoneal tumors: imaging features with pathologic correlation. Radiographics 2008; 28: 583-607.
27. Bevan KE, Mohamed F, Moran BJ. Pseudomyxoma peritonei. World J Gastrointest Oncol 2010; 2: 44-50.

28. Oei TN, Jagannathan JP, Ramaiya N, et al. Peritoneal sarcomatosis versus peritoneal carcinomatosis: imaging findings at MDCT. AJR Am J Roentgenol 2010; 195: W229-235.

29. Pickhardt PJ, Fisher AJ, Balfe DM, et al. Desmoplastic small round cell tumor of the abdomen: radiologic-histopathologic correlation. Radiology 1999; 210: 633-638.

30. Ake AC, Menzli A, Lecomte JC, et al. Peritoneal splenosis mimicking peritoneal carcinomatosis: a case report. Diagn Interv Imaging 2012; 93: 890-893.

31. Chang ES, Bachul P, Szura M, et al. Peritoneal “melanosis". Pol J Pathol 2015; 66: 330-333.

32. Machado NO. Sclerosing encapsulating peritonitis: review. Sultan Qaboos Univ Med J 2016; 16: e142-151.

33. Kamaya A, Federle MP, Desser TS. Imaging manifestations of abdominal fat necrosis and its mimics. Radiographics 2011; 31: 2021-2034.

34. Tempany CM, Zou KH, Silverman SG, et al. Staging of advanced ovarian cancer: comparison of imaging modalities - report from the Radiological Diagnostic Oncology Group. Radiology 2000; 215: 761-767.

35. Low RN, Barone RM, Lucero J. Comparison of MRI and CT for predicting the Peritoneal Cancer Index (PCI) preoperatively in patients being considered for cytoreductive surgical procedures. Ann Surg Oncol 2015; 22: 1708-1715.

36. Bozkurt M, Doganay S, Kantarci M, et al. Comparison of peritoneal tumor imaging using conventional MR imaging and diffusion-weighted MR imaging with different $b$ values. Eur J Radiol 2011; 80: 224-228.

37. Michielsen K, Vergote I, Op de Beeck K, et al. Whole-body MRI with diffusion-weighted sequence for staging of patients with suspected ovarian cancer: a clinical feasibility study in comparison to CT and FDG-PET/CT. Eur Radiol 2014; 24: 889-901.

38. An H, Lee EYP, Chiu K, Chang C. The emerging roles of functional imaging in ovarian cancer with peritoneal carcinomatosis. Clin Radiol 2018; 73: 597-609. 